\title{
Student Perspectives in Quantum Physics
}

\author{
Charles Baily and Noah D. Finkelstein
}

Department of Physics, University of Colorado, Boulder, Colorado 80309, USA

\begin{abstract}
Introductory courses in classical physics are promoting in students a realist perspective, made up in part by the belief that all physical properties of a system can be simultaneously specified, and thus determined at all future times. Such a perspective can be problematic for introductory quantum physics students, who must develop new framings of epistemic and ontological resources in order to properly interpret what it means to have knowledge of quantum systems. We document this evolution in student thinking in part through pre/post instruction evaluations using the CLASS attitude survey.[1] We further characterize variations in student epistemic and ontological commitments by examining responses to an essay question, coupled with responses to supplemental quantum attitude statements. We find that, after instruction in modern physics, many students are still exhibiting a realist perspective in contexts where a quantum perspective is needed. We also find that this effect can be significantly influenced by instruction, where we observe variations for courses with differing learning goals.
\end{abstract}

Keywords: Physics education research, quantum mechanics, measurement, PhET simulations.

PACS: 01.40.Fk, 03.64.-W

\section{INTRODUCTION}

There are good reasons to believe that introductory courses in classical physics are promoting in students a perspective that we call local realism. A realist perspective would be deterministic, where all physical quantities describing a system can be simultaneously specified for all times. For example, students in classical electrodynamics courses are typically instructed to think of an electron as a localized particle with a well-defined position and momentum. Having had their commitment to a realist perspective reinforced through prior instruction may be problematic for students of modern physics, who must then learn that physical observables are indeterminate outside the context of measurement, and subject to the laws of probability.[2] From a quantum perspective, an electron's position and momentum cannot be simultaneously specified; and while it is sometimes appropriate to model an electron as a localized particle, at other times it must be thought of as a delocalized wave.

We are therefore concerned with how students' perspectives change as they make the transition from learning classical physics to learning quantum physics. An analysis of student responses to pre/post surveys at various stages of instruction allows us to infer the development and reinforcement of a realist perspective in classical physics students, as well as the emergence of a quantum perspective in students as they progress through a course in modern physics. Additional responses to an end-of-term essay question, coupled with responses to supplemental quantum attitude statements, show that a student's degree of commitment to either a realist or a quantum perspective is not necessarily robust across contexts.[3] And so, although students might demonstrate a quantum perspective when discussing an electron diffraction experiment, they may at the same time be exhibiting a realist perspective when suggesting that an electron in an atom can have a definite (but unknown) position at all times. We further find, through a comparison of two recent semesters of a modern physics course offered at the University of Colorado, that this effect can be significantly influenced by instruction. We conclude from the available data that specific attention paid to the ontological interpretation of quantum processes during instruction may aid in the cultivation in students of a suitable quantum perspective.

\section{DATA SOURCES AND RESULTS}

The University of Colorado (CU) offers a threesemester sequence of calculus-based introductory physics: PHYS 1110 and 1120 are large-lecture courses $(\mathrm{N} \sim 300-600)$ in classical mechanics and electrodynamics; PHYS 2130 and 2170 are two independent courses in modern physics, the former designed for engineers and the latter intended for 
physics majors, each with a typical class size of $\sim 75$ students ${ }^{1}$.

The Colorado Learning Attitudes about Science Survey[1] (CLASS) was given at the beginning and end of the semester to students from each of these courses as a means of characterizing their beliefs about physics and the nature of learning physics. Students responded using a 5-point Likert scale (indicating from strong disagreement to strong agreement) to a series of statements, including \#41: "It is possible for physicists to carefully perform the same experiment and get two very different results that are both correct." Responses to this question are not scored as being "expert-like" or not because there is no consensus among experts as to how to respond; the statement's ambiguities allow for a number of legitimate interpretations to emerge when formulating a response.

There is a clear trend in how student responses to statement \#41 change over the course of the introductory sequence (Figure 1). Among students starting off in PHYS 1110, many more will agree with this statement than disagree; yet the number in agreement decreases significantly during introductory classical physics instruction, while an increasing number of students disagree. This trend then reverses itself over a single semester of modern physics, with an even greater percentage of students agreeing with the statement than at the beginning of classical physics instruction.

To clarify students' interpretation of the statement and reasoning behind their responses to $\# 41$, an optional text box has been added to the online CLASS survey. An examination of the types of concepts invoked by students in their responses provides some insight into the types of resources that are available to students at various stages of instruction. Open-ended

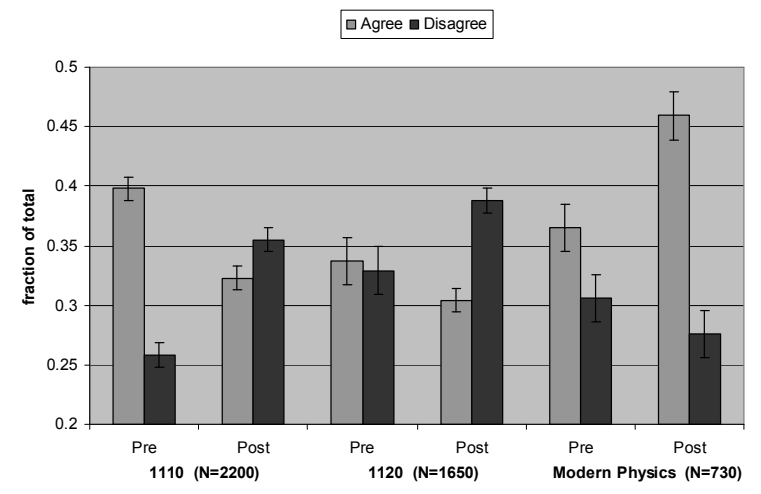

FIGURE 1. Aggregate data for student responses to CLASS statement \#41 across a three semester introductory physics sequence. Error bars represent the standard error on the mean.

\footnotetext{
${ }^{1}$ Unless indicated, data from the two modern physics courses have been combined for the purposes of this discussion.
}

TABLE 1. Categorization of concepts invoked by students in response to CLASS \#41.

\begin{tabular}{cl}
\hline A & $\begin{array}{l}\text { Quantum Theory/Phenomena } \\
\text { Relativity/Different Frames of Reference }\end{array}$ \\
\hline \multirow{2}{*}{ B } & $\begin{array}{l}\text { There can be more than one correct answer. } \\
\text { Experimental results are open to interpretation. }\end{array}$ \\
\hline C & $\begin{array}{l}\text { Experimental/Random/Human Error } \\
\text { Hidden Variables, Chaotic Systems }\end{array}$ \\
\hline \multirow{2}{*}{ D } & $\begin{array}{l}\text { There can be only one correct answer. } \\
\text { Experimental results should be repeatable. }\end{array}$ \\
\hline
\end{tabular}

responses were coded into four categories through an emergent coding scheme.[4] (Table 1)

Our analysis shows that, prior to instruction in modern physics, $59 \%$ of those who agreed with statement \#41 offered category $\mathrm{C}$ explanations, while category $\mathrm{D}$ was preferred by those who disagreed $(69 \%)$. As to whether the group of students who provided reasoning for their answers is representative of the group of all respondents, we do find that Neutral respondents are less likely to provide their reasoning, and that those in agreement are more likely than others to provide reasons for their answers. However, we see through a comparison of final course grades that the group of students who agreed with \#41 and provided their reasoning is not statistically different from the group who agreed but provided no reasoning for their response - and likewise for Neutral and Disagree respondents. We therefore conclude that, among introductory physics students, those who disagree with \#41 primarily concern themselves with the idea that physics is deterministic, while those who agree with the statement are more conscious of the possibility for random, hidden variables to influence the outcomes of two otherwise identical experiments.

Categories B, C, and D are collapsed in Table 2 into a single category representing reasoning that does not invoke quantum or relativistic phenomena. As would be expected, few students invoke quantum phenomena when responding before any formal instruction in modern physics, while a single semester of modern physics results in a four-fold increase in the percentage of students who believe that quantum or relativistic phenomena could allow for two valid experimental results to disagree - as well as a 30 point increase in the percentage of students who invoked

TABLE 2. Distribution of reasoning invoked by students in response to CLASS \#41.

\begin{tabular}{c|cccc}
\hline \multicolumn{5}{c}{ Before Instruction in Modern Physics } \\
\hline $\mathbf{( N = 5 0 7 )}$ & Disagree & Neutral & Agree & Total \\
A & $2 \%$ & $1 \%$ & $6 \%$ & $9 \%$ \\
B-D & $37 \%$ & $14 \%$ & $40 \%$ & $91 \%$ \\
\hline \multicolumn{5}{c}{ After Instruction in Modern Physics } \\
\hline $\mathbf{N}=\mathbf{8 3})$ & Disagree & Neutral & Agree & Total \\
A & $10 \%$ & $5 \%$ & $24 \%$ & $39 \%$ \\
B-D & $22 \%$ & $14 \%$ & $25 \%$ & $61 \%$ \\
\hline
\end{tabular}


quantum or relativistic phenomena in their reasoning, regardless of their Likert-scale response.

Further data have been collected from two recent semesters of PHYS 2170 in order to characterize how students' commitments to either a realist or quantum perspective can vary by context, and to see if these commitments can be influenced by different types of instruction and learning goals. Course 2170A was taught by a Physics Education Research (PER) instructor who employed in-class research-based reforms, including PhET simulations[5] designed to provide students with a visualization of quantum processes. Course $2170 \mathrm{~B}$ was taught the following semester in the form of traditional lectures delivered from a chalkboard. A typical semester of modern physics at $\mathrm{CU}$ devotes roughly one-third of the lectures to special relativity, with the remaining lectures covering the foundations of quantum mechanics and simple applications.

Students from both of these courses were given an end-of-term essay question asking them to argue for or against statements made by three fictional students who are discussing the representation of an electron in the Quantum Wave Interference PhET simulation.[6] In this simulation a single blob (representing the probability density) emerges from an electron gun, passes through two slits, and then a small dot appears on a detection screen; after a long time (many electrons) an interference pattern develops. (See Appendix for the full text of this question.) Each statement made by a fictional student is intended to represent a potential perspective on how to model the electron between the time it is emitted from the electron gun and when it is detected at the screen. Responses were coded according to whether students preferred a realist or a quantum perspective in their argumentation; the following two student quotes are exemplary of the types of responses seen:

Realist: "We just can't know EXACTLY where the electron is and thus the blob actually represents the probability density of that electron. In the end, only a single dot appears on the screen, thus the electron, wherever it was in the probability density cloud, traveled in its own direction to where it ended up."

Quantum: "The blob is the electron and an electron is a wave packet that will spread out over time. The electron acts as a wave and will go through both slits and interfere with itself. This is why a distinct interference pattern will show up on the screen after shooting out electrons for a period of time."

The distribution of all responses for the two courses is summarized in Table 3; columns do not add to $100 \%$ since some students provided a mixed or otherwise
TABLE 3. Student response to an end-of-term essay question from two recent semesters of PHYS 2170.

\begin{tabular}{c|cc}
\hline & 2170A & 2170B \\
\hline Realist & $18 \%$ & $75 \%$ \\
Quantum & $78 \%$ & $11 \%$ \\
\hline
\end{tabular}

unclassifiable response. There is a strong bias towards a quantum perspective among $2170 \mathrm{~A}$ students, while students from 2170B highly preferred a realist perspective.

Students from both courses also responded at the beginning and end of the semester to additional survey statements appended to the CLASS for modern physics students, including QA\#16: "An electron in an atom has a definite but unknown position at each moment in time." It might be expected that a student who has learned to view an electron as being delocalized in space in the context of an electron diffraction experiment should also see it as such when considering whether an electron in an atom can have a definite position in the absence of measurement.

TABLE 4. Student responses to QA\#16: "An electron in an atom has a definite but unknown position at each moment in time."

\begin{tabular}{c|cc|cc}
\hline \multicolumn{2}{|c|}{ 2170A } & \multicolumn{2}{c}{ 2170B } \\
\hline & PRE & POST & PRE & POST \\
Disagree & $22 \%$ & $44 \%$ & $10 \%$ & $23 \%$ \\
Neutral & $32 \%$ & $17 \%$ & $39 \%$ & $21 \%$ \\
Agree & $44 \%$ & $39 \%$ & $48 \%$ & $54 \%$ \\
\hline
\end{tabular}

Instead, the data contained in Table $\mathbf{4}$ do not show the same bias toward a single perspective as in Table 3. From a quantum perspective, disagreement with QA\#16 can be characterized as favorable; Table 4 shows that students in $2170 \mathrm{~A}$ posted a 22 point increase in favorable responses, and those from 2170B posted a $13 \%$ favorable shift; but while 2170 A showed a $5 \%$ decrease in unfavorable responses, 2170B students increased their unfavorable responses at the end of the semester by 6 percentage points. The postdata in Table 4 from the two courses can be combined and presented in another format, by grouping postresponses to QA\#16 according to how those same students responded to the essay question. (Table 5) Here, we see that students who had preferred a quantum perspective tended to answer QA\#16 favorably, while the majority of students who preferred a realist perspective chose an unfavorable response.

TABLE 5. Post-responses to QA\#16, grouped according to student responses to the end-of-term essay question.

\begin{tabular}{c|cccc}
\hline & Disagree & Neutral & Agree & Total \\
\hline Quantum & $56 \%$ & $11 \%$ & $33 \%$ & 100 \\
Realist & $18 \%$ & $18 \%$ & $64 \%$ & 100 \\
\hline
\end{tabular}




\section{DISCUSSION AND CONCLUSIONS}

We believe there is sufficient evidence to infer the development and reinforcement of a realist perspective in many students as a result of instruction in classical physics. Such a perspective can be viewed within a resources framework[7] as dynamic, emerging in a given context in the minds of students from the coordinated activation of finer-grained resources. The framing of these resources determines what types of knowledge is relevant (e.g. what kinds of knowledge can be had about a physical system, and thus how to formulate a representation of that system). We find that, after instruction in modern physics, many students are bringing quantum-related resources to bear when formulating their responses to statement $\# 41$; the activation of these resources led to a reinterpretation of the statement from a new perspective, resulting in a significant shift in student responses after a single semester of modern physics.

The representation of an electron as a delocalized wave in the absence of a position measurement cannot be considered to be a stable concept among introductory modern physics students. Instead, we see that how those students view an electron can vary according to how the question is posed. Most interesting is the pronounced difference in responses to the essay question between two offerings of the same modern physics course. The potential reasons for these differences are too myriad to identify completely, though informal interviews with the instructors revealed a difference in learning goals when it came to interpretations of quantum processes. By providing a visualization of the electron in a diffraction experiment via the PhET simulation, Instructor A paid specific attention to students' mental models and interpretations of quantum measurements. It is worth noting that the simulation equates the probability density with the electron itself, which may be controversial among the physics community. Instructor B felt the question of what the electron is "doing" between when it is emitted from the gun and when it is detected to be philosophical in nature, still open to debate among physicists, and not necessarily relevant to student understanding and application of the mathematical formalisms of quantum mechanics.

Although the results contained in this study are preliminary, it seems evident that an instructor's choice of learning goals can have a demonstrable impact on student learning. And while the available data appear to support the usefulness of emphasizing ontological interpretations of quantum processes, we believe that student attitudes and beliefs are important in their own right. We also note that previous studies have shown student beliefs about physics to be correlated not only with self-reported student interest,[8] but also with conceptual understanding.[9]

\section{APPENDIX}

\section{Full Text of Essay Question:}

Three students discuss the Quantum Wave Interference simulation, in which a blob emerges from an electron gun, goes through two slits, and then a small dot appears on the screen, which is recognized as a "hit" of the electron. After a long time (many electrons) an interference pattern of "hits" is observed on the screen.

Student 1: That blob represents the probability density, so it tells you the probability of where the electron could have been before it hit the screen. We don't know where it was in that blob, but it must have actually been a tiny particle that was traveling in the direction it ended up, somewhere within that blob.

Student 2: No, the electron isn't inside the blob, the blob represents the electron! It's not just that we don't know where it is, but that it isn't in any one place. It's really spread out over that large area up until it hits the screen.

Student 3: Quantum mechanics says we'll never know for certain, so you can't ever say anything at all about where the electron is before it hits the screen.

Which students (if any) do you agree with, and why? What's wrong with the other students' arguments? What is the evidence that supports your answer?

\section{ACKNOWLEDGMENTS}

The authors wish to thank the CU physics faculty members who helped facilitate this research, as well as Sam McKagan for thoughtful discussions and suggestions, and the rest of the Physics Education Research group at $\mathrm{CU}$ for their helpful insights. Supported in part by NSF CAREER Grant No. 0448176; findings are those of the authors.

\section{REFERENCES}

1. W. K. Adams, et al. PRST: PER 2, 1, 010101 (2006).

2. R. D. Knight, Physics for Scientists and Engineers, Pearson Education, Inc., 2004, p. 1253 et seq.

3. N. D. Finkelstein, Int. J. Sci. Educ., 27, 1187 (2005).

4. J. W. Creswell, Education Research, $2^{\text {nd }} E d$., Prentice Hall, 2005, pp. 397-8.

5. S. B. McKagan, et al. Am. J. Phys. 76, 406 (2008)

6. http://phet.colorado.edu/simulations/sims.php?sim=qwi

7. D. Hammer, et al. "Resources, Framing and Transfer" in Transfer of Learning, edited by J. Mestre, Information Age Publishing, 2005, pp. 89-119.

8. K. K. Perkins, et al. PERC Proceedings 2005 p.137

9. K. K. Perkins, et al. PERC Proceedings 2004 p. 61 